\title{
The making and evaluation of Picts and Pixels: Mixed exhibiting in the real and the unreal
}

\author{
Catherine Anne Cassidy ${ }^{1}$, Adeola Fabola ${ }^{1}$, Elizabeth Rhodes $^{1}$ and A Miller ${ }^{1}$ \\ School of Computer Science, Universitiy of St Andrews, United Kingdom \\ ahr1@st-andrews.ac.uk \\ http://www.cs.st-andrews.ac.uk/
}

\begin{abstract}
Museums publicly display collections in a physical space to relay narratives and concepts to their audiences. Progressive technologies in an exhibition can bring in varying demographics and gather higher footfall for a museum as well as present digital heritage interpretation in an innovative manner. A mixed media exhibition can facilitate subjects with limited physical resources or difficult to display pieces as well as the visual landscape the objects were found within. A combination of Virtual Reality headsets, 3D digitized objects, digitally reconstructed archaeological sites alongside traditional object displays as methods of interpretation substantiate research in techniques and usability as well as challenges of recoup cost and digital literacies. This paper investigates the methodology, technology and evaluation of the mixed media exhibition Picts \& Pixels presented by Culture Perth and Kinross and the Open Virtual Worlds research team at the University of St Andrews at the Perth Museum and Art Gallery in summer 2017.
\end{abstract}

Keywords: Virtual Reality, Mixed Reality, digital exhibits, Picts

\section{Introduction}

Gildas, a 6th century Romano-British monk chronicled the Pictish people as "butchers" with "villainous faces" [1]]. Herodian wrote they were "ignorant of the use clothes" which was only to display their tattooed bodies in battle [1]]. How academics view the Picts has changed since Gildas and Herodian, however in modern media do these predisposed assumptions still preside. The lure to the mysterious Picts is just that, the mystery that encircles them. For a society to have prevalence in Scotland since the Iron Age, territory spanning north of Edinburgh, reaching the Highlands and Islands of Scotland to leave only a few remnants of their existence is cryptic and enticing for the dramatists. The evidence left of their world, however little, is intricate, sophisticated and rich. Over one hundred and fifty stone sculptures found within Scotland show the incredible intricacies of geometric and symbolic work as well as some of the "most naturalistic animal sculptures...in Europe" [2]].

Exhibiting the Picts through artefacts and the narrative of their known history, tells people what is known about them and shows what is held within a museum's stores. However, a large amount of the Picts story is within the landscape, from standing stones to hillforts, which cannot be brought physically back into an exhibition space.

This paper assembles the innovative digital work created by the Open Virtual Worlds research group at the University of St Andrews for an exhibition at the Perth Museum and Art Gallery entitled Picts and Pixels. The exhibition conatins 
multimodal digital exhibits with the physical presentation of artefacts and a soundscape to create an immersive space to stimulate interest and learning about the Pictish contribution to Scottish history. The exhibition was accompanied by a program of public events including workshops, a museum late event and a series of expert talks.

The exhibition brought together digital reconstructions based upon archaeological excavation, aerial photography of historic sites, digital artefacts produced from photogrammetry, light and laser scanning methods as well as spherical technologies alongside and in combination with physical artefacts. This mixture of technology and archaeological finds were brought together in the main gallery hall of the museum as a temporary exhibit for four months in the summer of 2017. The exhibition attracted thousands of visitors and a considerable amount of media attention, including a feature in the Guardian's Top Tens: Your At A Glance Guide to the Best Culture This Week feature.

This paper discusses related work prior to the exhibit as well as the background and methodology of the content and outputs created. We then present a description of the exhibition as it appeared to visitors and analyse the technology behind each of the interactive displays. Evaluation of the exhibition took the form of questionnaires and interviews of both experts connected with the museum and visitors to the exhibition.

\section{Related Work and Background}

The technology, experimentation and digital design for the exhibition builds upon a program of work exploring the digital representation of heritage. This has involved collaborations with museums, academics, archaeologists and historians in developing digital cultural heritage outputs, which enable cross reality exploration inside and in landscapes, immersive exhibits, the application of game technology and exploration with mobile applications. The curatorial team involved with the exhibition at the Perth Museum and Art Gallery drew upon their experience with a previous digital exhibit that had been especially successful.

Digital reconstructions have been developed based upon historical and archaeological evidence. This includes the reconstructions of a 6th Century Spartan Basilica [89]], St Andrews Cathedral as it would have stood in the 13th century [[14]], the Brora Salt Pans based upon community excavations [[14] and the Edinburgh cityscape as it would have been in 1544 [[16]]. All were deployed in various digital formats for discovery and exploration, from geo-location aware mobile applications, first person world exploration in virtual reality and spherical tours online. The collaboration between the University of St Andrews and the Timespan Museum and Art Gallery has developed a suite of digital interpretation [11] of historic content. This includes an immersive 3D interactive room [17]], a digital trail mobile application [11] and virtual cross reality workstations [[10]]. Investigation of cross reality exploration of historic sites addressed the relative benefits of fixed point and free exploration of digital scenes that represented the past.

As part of the Horizon 2020 EU-LAC MUSEUMS project, workflows for photogrammetry to create $3 \mathrm{D}$ models for use in museum settings were developed 
and made efficient. These included workflows for using photogrammetry to create 3D models using commodity equipment for community museums as well as a system for creating virtual tours, linking together spherical images of scenes and embedding interpretation within them.

The work described in this paper brings together aspects of the above work, within the context of a single exhibition. Further it combines the digital representation with physical exhibits creating mixed reality exhibition.

The technology, experimentation and digital design for the exhibition builds upon the experience the Open Virtual Worlds (OVW) team has in developing digital cultural heritage outputs, such as virtual museums, interactive exhibits and exploration mobile applications. The team involved with the exhibition at the Perth Museum and Art Gallery drew upon their experience with a previous digital exhibit that had been especially successful.

\section{History}

After the Romans left Britain, the area around the River Tay in Perthshire came under Pictish influence. The Picts were a Celtic people who spoke a language similar to Welsh. Many place-names in the Tay valley contain Pictish elements. For example, the name Perth possibly comes from the Pictish 'pert', meaning woodland.

The Picts created elaborate art, including large carved stones, a number of which can be seen in the countryside around the Tay and River Earn, while others have been moved to local museums. At some point (probably before the end of the seventh century A.D.) the Picts converted to Christianity. Churches and monasteries were established, often at places which already had religious or political significance, such as Forteviot and Abernethy.

The Pictish period also saw continuing, or in some cases renewed, use of hillforts. Forts at Abernethy, Moredun Top (on Moncreiffe Hill near Perth), and several other sites, seem to have been occupied during the Early Middle Ages. In 728 A.D. Moncreiffe Hill may have been the scene of a major battle, when the Pictish king engus defeated his rival Ailpn in a struggle for control of southern Pictland.

During the ninth century the Picts joined with the Scots (a Gaelic speaking people based in Argyll) to create a new kingdom called Alba. This union is often seen as the beginning of the modern nation of Scotland. The Tay and Earn valleys were at the heart of the new kingdom. There was a royal palace at Forteviot, where one of the first kings of Alba, Cinaed mac Ailpn, died in 858 A.D. From around the beginning of the tenth century onwards the kings of Alba, and later Scotland, were inaugurated at Scone, probably on the mound known as Moot Hill.

\section{Methodology}

As part of the Tay Landscape Partnership, a larger funded project OVW was a partner of, the research group was approached to collaborate with Culture Perth and Kinross (CPK) for a forthcoming exhibition at the Perth Museum and Art Gallery, Scotland. Technology and its potential for the exhibition was outlined 


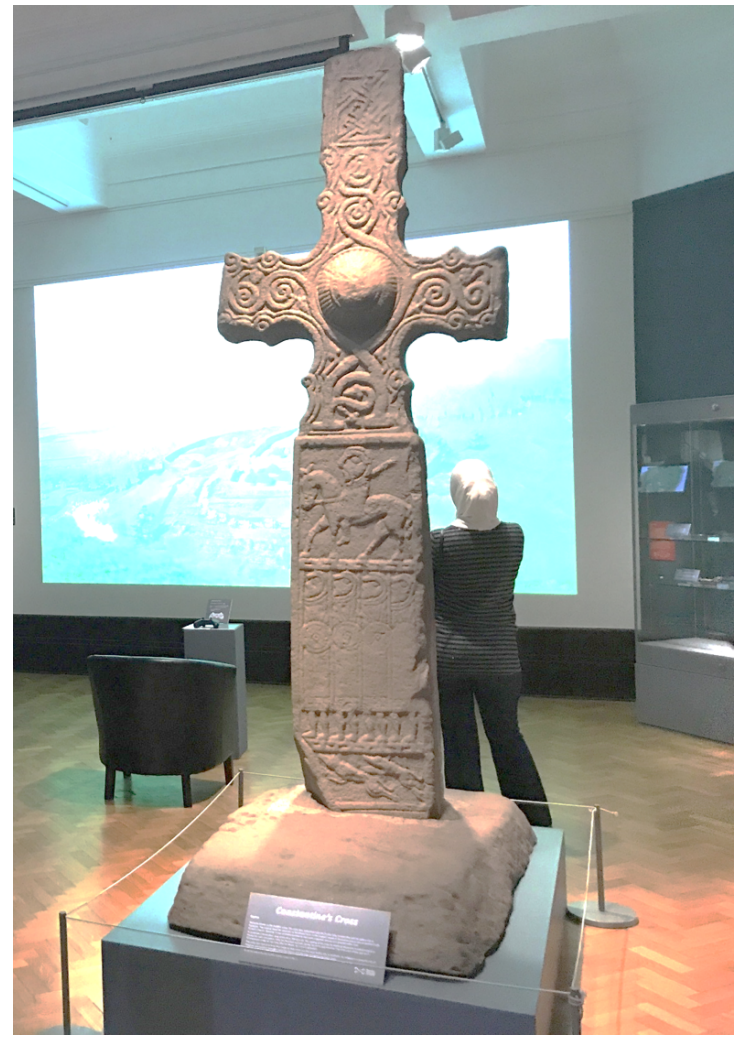

Fig. 1: Combining physical artefacts with digital representations in a museum space

and included in funding applications early in the exhibition's planning stages. All meetings, whether discussing conceptual, visual or thematic ideas, members of OVW participated.

The museum chose the Picts as a subject due to their historical prevalence in the local area, the unusually robust collection the Perth Museum had within its stores as well as the general mysteriousness the Picts tend to manifest. Objects to feature were first decided by the exhibition planning committee, while the method of displaying the digital heritage evolved over the course of exhibition development. Plans already included developing a virtual reconstruction of two archaeological sites, Moredun Top and Forteviot, both active fieldwork locations at different points during the Tay Landscape Partnership.

The logic behind including technology side by side with the physical artefacts in the museum (fig 1) was to highlight the areas that could be disseminated about the Picts but could not be actually properly presented in a traditional manner within the gallery. These reasons included: 1) Difficulty of displaying stone objects where all surfaces of the object could be publicly viewed, leading to key markings or symbols to be missed 2), archaeological sites related to the TLP project and exhibition that had sufficient data to be digitally reconstructed in a virtual environment, but were part of the modern landscape. 3) To feature a large standing stone, the St Madoes stone, displayed in the entryway of the museum but whose movement into the gallery for the exhibition would have been imprac- 


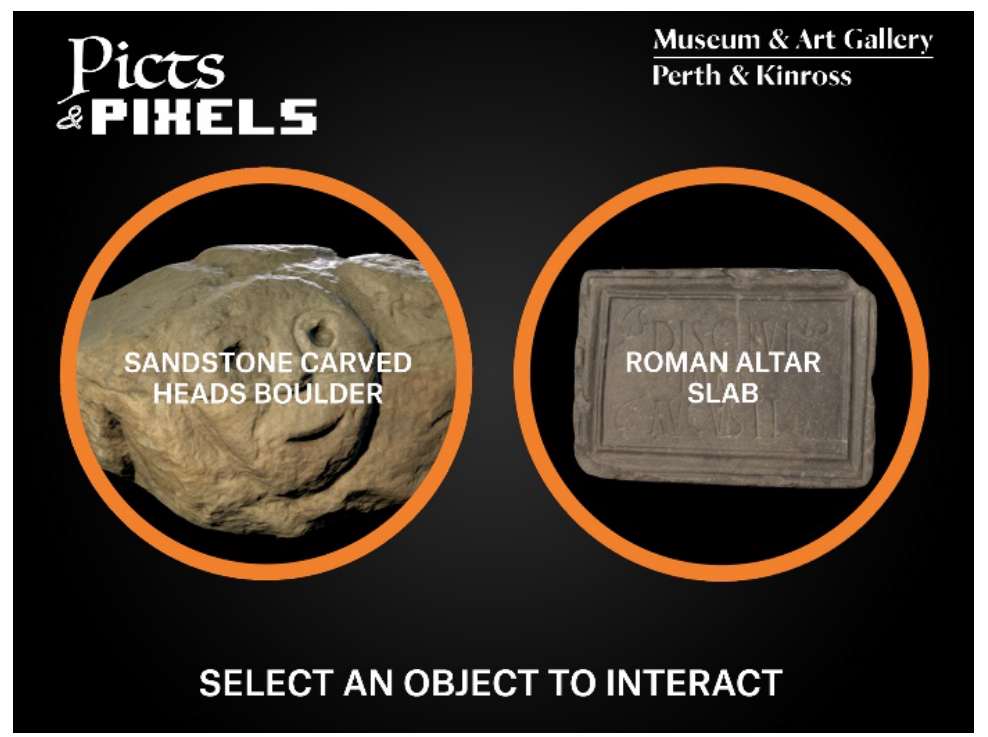

Fig. 2: Menu of 3D Objects

tical due to its weight. 4) Highlight the historically probable coloured painting that would have originally been on the St Madoes stone, but has since deteriorated. 5) Assist in increasing the number of displays within a large gallery space amongst physical objects on display from Perth and National Museum Scotland (NMS). 6) Test the capacity of latest technology for adaptable and innovative use in a full museum exhibition. 7) Include exhibits and host workshops that helped bring in a continually low demographic within the museum (under 20 years of age).

\section{The Exhibition}

The goals of the exhibition were to: 1) introduce an Ancient Roots theme to the public in an engaging way; 2) provide an opportunity for tourists to learn about Perth's place in the Pictish period, and Pictish culture, through a range of interactives and interpretation; 3 ) provide opportunities for audiences to learn about Pictish heritage on their doorstep; 4) deliver an exhibition that enables a programme of activities that contribute to the Year of History, Heritage and Archaeology; 5) showcase Perth Museum collections, augmented by key loansin from NMS; 6) increase staff knowledge in preparation for new displays and development of Ancient Roots themes.

The exhibition was held in the main gallery hall in May, June, July and August of 2017. As a visitor approached the entrance to the exhibition, they would see the full length of the dimly lit hall through a stone arch portal. A cast of a large Pictish cross was silhouetted against a moving projection of Moredun Top landscape. A soundscape of appropriate natural sounds, birdsong, wind and water interspersed with a Pictish bell tolling periodically set the atmosphere. The exhibition provided a framework around which workshops and public events were organised. These included a series of public lunchtime lectures, weekend 


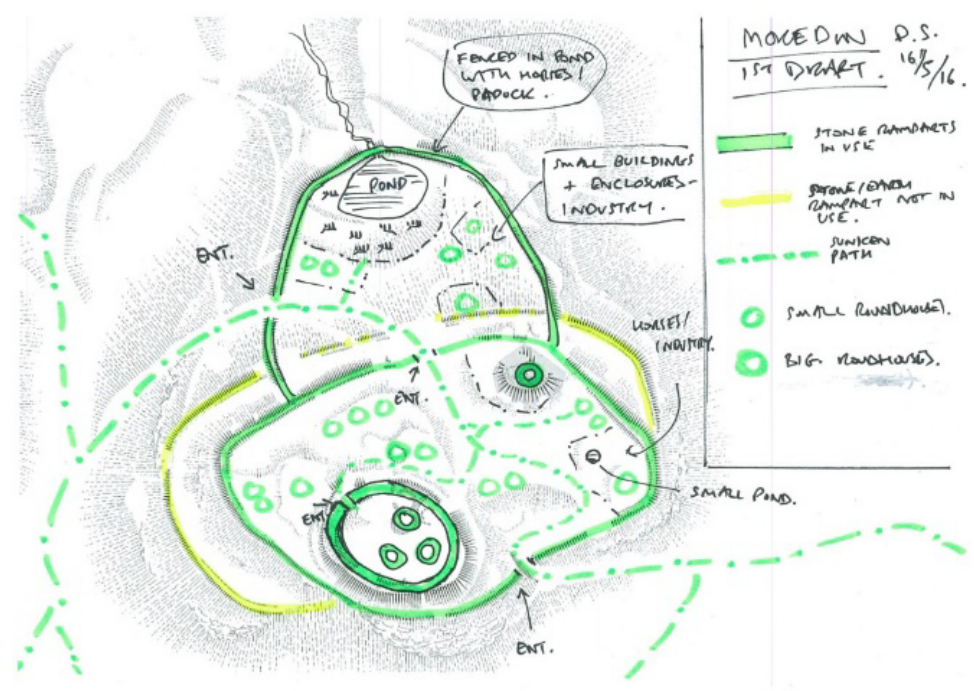

Fig. 3: Sketch of Moredon Top features

workshops aimed at teaching school students on how to make virtual tours, a Museum Late event featuring digital interpretation as well as a technology open day featuring digital games and exploration. The open day towards the end of the exhibition, part of the Perth Medieval Festival, attracted over 500 visitors.

\subsection{Modes of Engagement and Interaction with the Exhibition}

In order to achieve the goal of introducing the exhibition's audiences to the theme of Ancient Roots in an engaging way, the interactive exhibits needed to provide a comprehensive style of learning and entertainment for all. Each exhibit was designed to enable visitors to gain a positive experience with or without having physical interaction. The projections of the reconstructions played videos on loops when the interactive went idle. The Virtual Museum exhibit's Oculus Rift view was mirrored to a monitor that enabled visitors to also view what the single user was exploring. The 3D digitized objects on tablets played a video of the selected object rotating when the digital object was not being manipulated. This design decision was aimed to both cater to those satisfied with passive engagement and to encourage further participants. Some visitors that did not engage directly with interactives, by using the VR headset or Xbox controllers, were asked their reasoning for evaluation purposes. Some cited a lack of confidence with VR and games, and contentment with watching the idle content. The inclusion of a screen which mirrors the Virtual Reality headset content also enabled group-based exploration for participants who visited with family and friends. Only one visitor could use the headset at a time, so the screen enabled the other members of the group to share the view, facilitating conversations between them. This is important because it helped visitor to develop shared views [[3]].

The projection interactive exhibits were controlled via an Xbox controller. This allowed users to move from idle video to photospheres and control the view within the photospheres. The X-Box controller is well understood by a high proportion of the population and designed to be easy to pick up and use. 


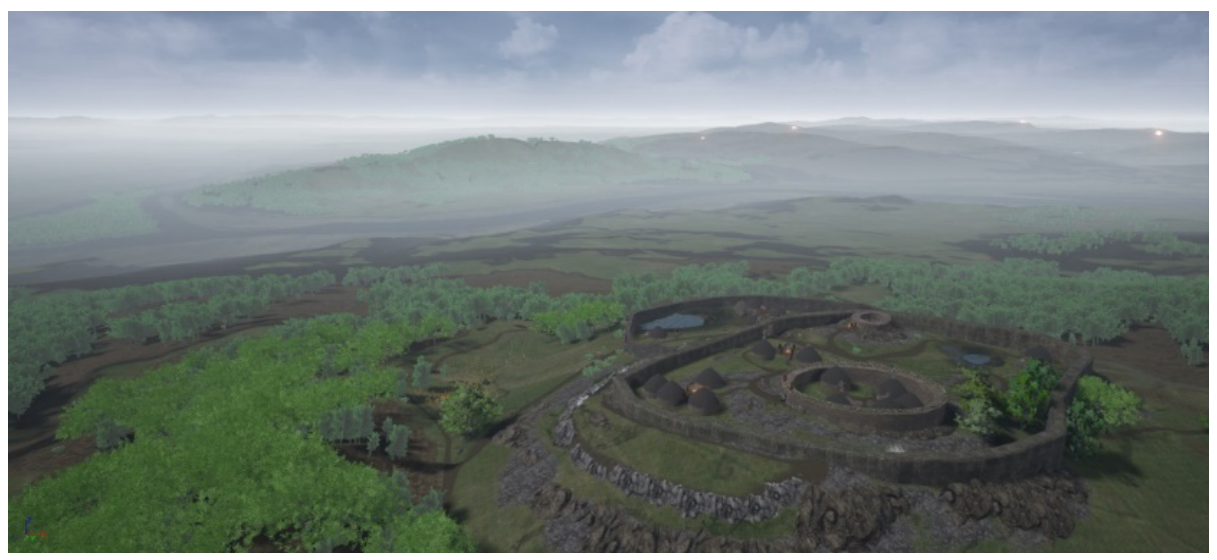

Fig. 4: View of Pictish Landscape

The Virtual Reality Headset also used an Xbox controller. This was largely unproblematic but did raise some issues. Given that the view of the virtual environment in the headset occludes the participants' view of the real world, users' typically did not see the controller while they have the headset on. This had implications, especially for users who are not familiar with game controllers, as it may deter them from engaging with digital exhibits with technology they are unfamiliar with. On one hand, this makes a case for controller-free exhibits which may appeal tosome members of the population but lack the added functionality required for complex interaction. Ultimately, the decision regarding which one to adopt should depend on the objectives of the exhibit and the target audience, thus it behooves museums to gather data about their target audience during the planning and execution phases of exhibits.

\subsection{Digital Exhibits in a Shared Space}

The OVW team provided seven digital interactive exhibits, along with audio soundscapes, with multiple modes of delivery for the exhibition. These included: 1) Digital Soundscape: provided atmosphere and a shared experience across both the physical and digital exhibits; 2) Virtual Museum Exhibit: Immersive virtual museum containing 3D gallery within virtual environments, using Oculus Rift headset and Xbox game controller; 3) Interactive Surface Exhibits: Three mounted iPads with 3D object menu for digital object manipulation; 4) Interactive Digital Projection: Moredun Top reconstruction in spherical tour with Xbox controller, projected onto large gallery wall. Looped aerial and reconstruction video played when interactive idle. Forteviot reconstruction in spherical tour with Xbox controller, projected onto side gallery wall. Slow spinning spherical images on loop when interactive idle; 5) Painted Stone Media: Digitized St Madoes stone with an overlay of historical accurate colours. Video of colours added to the stone on loop.

Digital Soundscape The soundscape was designed to envelop the visitor's senses and to help them imagine stepping back through time. Natural Scottish woodland sounds, complete with bird calls, wind through rustling trees and a 
distant sound of a river provided the outdoor setting. A low hum of Gregorianlike chanting created an eerie and mysterious ambiance. A recording of a replica Pictish bell chimed every ten minutes, enough time where a visitor might hear it twice or three times while touring the exhibition. Audio was played on a surround sound system pre-installed in the gallery space. Levels were adjusted so that the front half of the partitioned gallery had slightly higher volume levels as to attract visitors in. As most of the interactive exhibits were in the rear partition of the gallery, more people were speaking in that section and the audio was lowered so it would not fight with groups engaging in the interactives.

Interactive Surface Exhibits An interactive digital surface enabled users to explore digital representations of Pictish stones and artefacts that they were mounted beside. As a popular and growing method of preservation and presentation [[7], the 3D models were placed to help with further interrogation. This facilitated visitors to view aspects of the objects that were either obscured in their physical presentation,or were so small minute details were barely visible. It also allowed visitors to manipulate the digital representation, zooming in on features, rotating and zooming out. This was achieved using a touch interface that many users are familiar with, as iPads were used.

A concise visual menu was designed (fig 2 that allowed the visitor to choose the object they wanted to interact with. A loading screen would show the rotating object before it settled onto the 3D object. The object could be fully rotated with zoom functionalities. An arrow button would take the visitor back to the main menu page to choose another object.

The system was a mobile application with simple navigation. 3D models were decimated to improve performance for the user. Models were captured by either photogrammetry techniques, laser scanning or structure light scanning and made into 3D meshes the 3D models with PhotoScan, VisualSFM and Meshlab.

Interactive Digital Projection This element of the exhibition set the tone and provided high-impact visuals for the exhibition as a whole. Reconstruction of the Moredun Top hillfort and surrounding area was undertaken in Unreal 4, enabling the hillfort to be located within its context. The landscape reconstruction stretches to all areas that can be seen from modern Moredun Top (fig 3). The reconstruction brought together data collected during three years of community archaeology excavations, with expert interpretation, landscape survey data including topology and the pollen record.

The first phase of the workflow was to establish the topology of the region. Data from OS and other surveys was merged in a GIS system and imported into the Unreal 4 engine where it was appropriately scaled. The landscape was populated with biologically and historically accurate flora and fauna. From the topology, likely types of regions were identified including hill top, marshland, steep slopes, arable land and grazing land. For each type a mix of plants and trees were defined and applied to the landscape. For the reconstruction of the hillfort, a scaled map of the features was created and imported into Unreal 4. This was scaled and positioned across the hilltop and provided a template for the creation of roundhouses, fortifications and pathways. These were then constructed based upon archaeological and historical evidence. Several rounds of reconstruction 


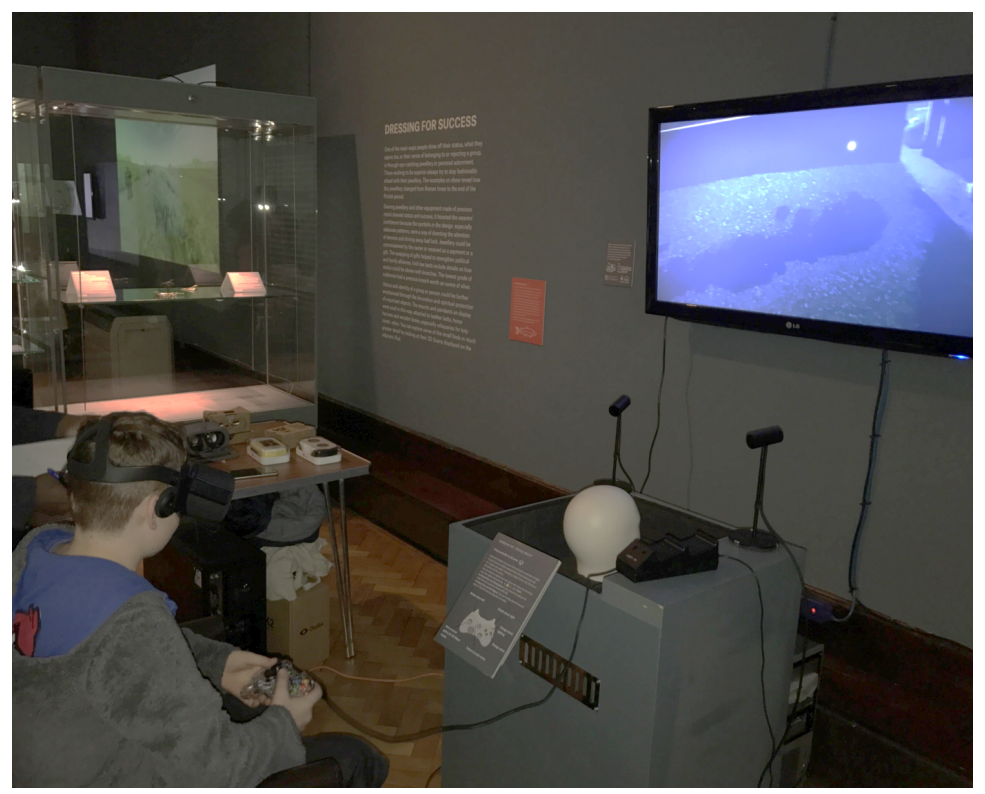

Fig. 5: A visitor interacting with the Picts \& Pixels exhibition

and expert critique were undertaken to ensure that the visualisation fitted with expert opinion (fig 4 ) .

The projection showed aerial footage of the Moredun Top site excavation in progress, different stages of the reconstruction process and the completed reconstruction. Short textual interpretation provides context. When interaction starts the exhibit jumps to a photosphere of the reconstruction. Visitors could control the view and jump to the photospheres showing aspects of the hillfort and landscape.

The exhibit was powered by a PC concealed in a custom cabinet which offered an instruction guide on its top and an Xbox controller. Interaction took place using the controller enabling navigation within photospheres, access to hotspots and movement between photospheres.

A second interactive exhibit of the Forteviot Pictish Burial ground was created using the same methodology and delivered using the same system type.

Virtual Museum Exhibit The free-form 3D environment of the exhibit was implemented in Unreal Engine 4. In addition, spherical media was extracted from these $3 \mathrm{D}$ environments, and were used to make up the scene components of the exhibit. The set-up allowed users to switch between an array of virtual environments either using game controllers or by triggering hotspots using head movements, and these provided the illusion of moving from one place to another, similar to a virtual tour. Digital representations of objects were imported as 3D meshes which were created using photogrammetry. A 3D gallery was used to house the digital artefacts, which was styled to place less emphasis on the level of detail in the virtual environment, and more emphasis on the

$3 \mathrm{D}$ artefacts. Controller-based interaction was built into the 3D gallery to allow users to rotate the artefacts around two axes, zoom in/out and move about 


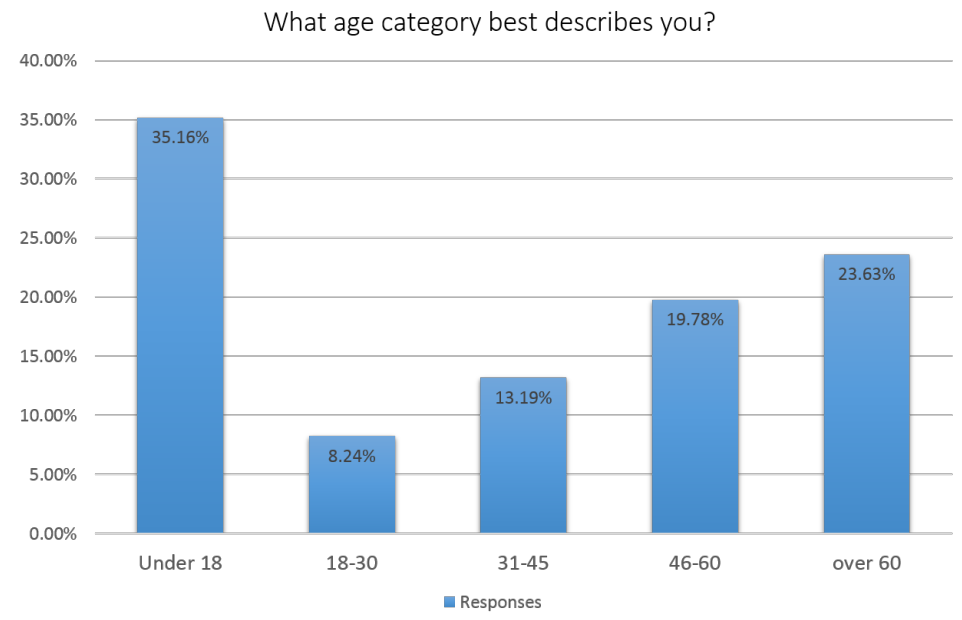

Fig. 6: Age distribution of the Picts \& Pixels exhibit visitors

the centrepiece. Users could also switch between an array of 3D artefacts in a manner that is similar to navigating through images in a photo gallery. In addition to representing scenes using spherical images and physical objects using 3D artefacts, the exhibit allowed for placing 3D artefacts in environments represented by spherical scenes. This was useful for associating scenes and objects thereby providing context and interpretation. All these components - spherical scenes, 3D object galleries, combined scenes and objects, and free-form 3D environments - were merged into an immersive, on-site museum installation with a top-level menu. Spherical scenes provided the ability to represent landscapes, cityscapes and geological structures. 3D artefacts provided the ability to represent artefacts, specimens and sculptures. Spherical scenes and 3D artefacts were combined to provide context and association between them. Audio and text were also combined with these media types to provide additional information and alternative means of content delivery (fig 5 ).

\section{Evaluation}

Throughout the entirety of the three-month long exhibition, a general evaluation was conducted by museum staff. The survey included age demographics, satisfaction with the exhibition and customers value for money.

\subsection{Exhibition run evaluation}

The exhibition was evaluated over a period of three months, during which 209 visitors provided feedback regarding their level of satisfaction and perceived value for money. 182 of the 209 provided information about their age group. Figure 7 shows that the under 18 visitors represented the largest age group with $35.16 \%$ of the total responders. Visitors aged over 60 represented the second largest group with $23.63 \%$ while visitors aged 46-60 represented the third largest group with $19.76 \%$. An evaluation exercise was conducted on the closing weekend of the 


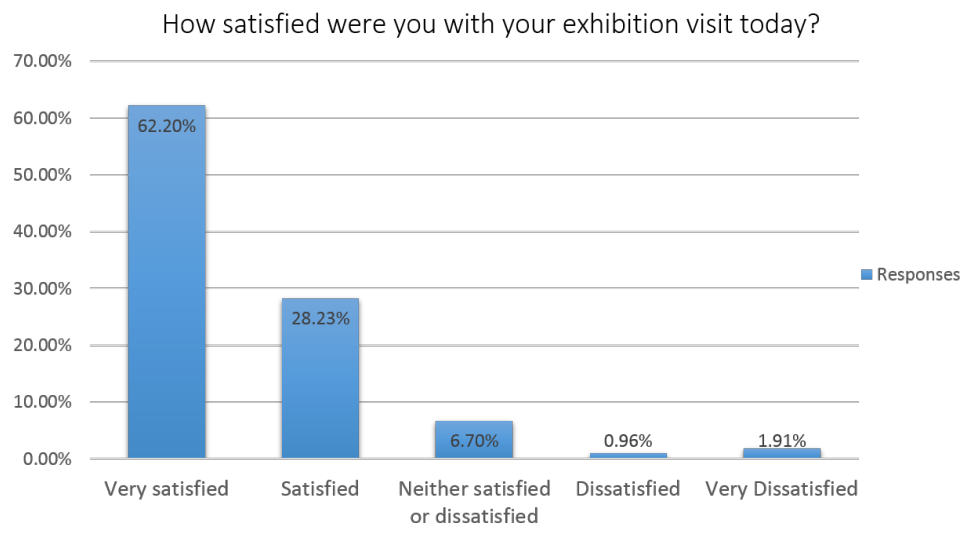

Fig. 7: Visitors' satisfaction for the Picts \& Pixels exhibit

exhibit. This was conducted during an open-day event, and participants were randomly selected from the larger group of museum visitors. Data was gathered from 22 of the 504 visitors recorded, through semi-structured interviews and Likert-scale questionnaire.

\subsection{Value and Impact}

Short written responses were noted on how visitors reacted when asked what they liked about the exhibition. 164 people responded. The general response was overwhelmingly positive with a focus on the virtual reality aspects but all aspects of the exhibit featuring, with special appreciation of the artefacts and the bells in the soundscape. As how visitors felt, the exhibit was able to create, "the feeling of being very close to these people who lived ordinary lives so long ago and shared a space which is so familiar to us many centuries later." It gave visitors the "chance to see what it would be like looking at the village itself, using the virtual reality headset." And it "helped me visualise what life would have been like." This feedback suggests the addition of interactive exhibits enabled visitors to feel close to and imagine what the past was like.

There were comments which found the "3D manipulation of objects and VR experience" enhanced the exhibit as well as "the 3D stuff was fab". And the VR headset was both an "absolutely fantastic way to immerse yourself with an artefact!" and good to "learn about the medieval houses on Moncrieff Hill." The combination of digital and physical worked well "3D images particularly enhanced the experience. Also, great variety of local artefacts." From the survey questionnaires of the 209 responders, $62.20 \%$ were very satisfied with the exhibition, $28 \%$ reported being satisfied, while less than $3 \%$ of the responders were not satisfied (fig 7). Over $82 \%$ of responders also felt that the exhibition provided value for money.

Participants at the open day evaluation filled out an experience questionnaire to evaluate whether the system is easy to use, whether they would recommend it for learning history, whether it has changed their perception of the subject matter, whether it has stimulated their interest in learning and whether they felt immersed in the virtual environment. The responses were gathered on a 5-point 


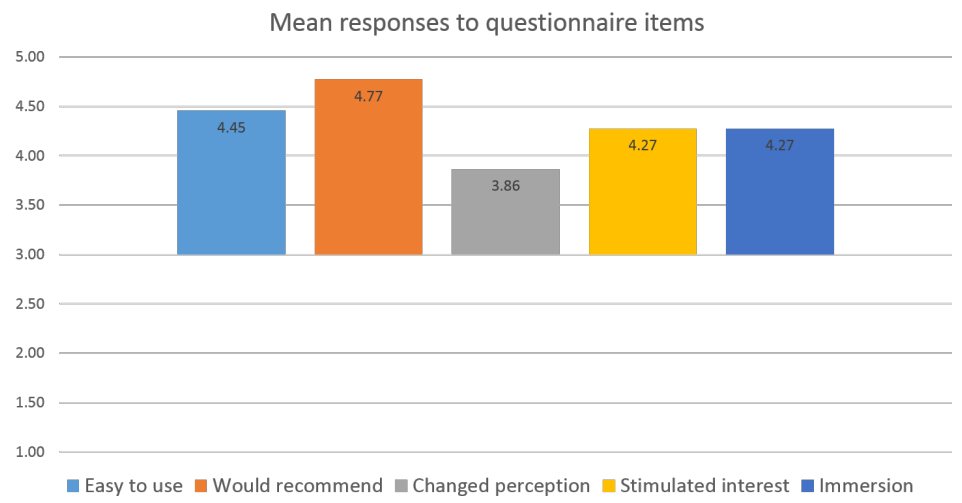

Fig. 8: Participants' mean responses to the Likert-scale user experience questionnaire

Likert-scale questionnaire and fig 8 shows that the mean responses to all the questionnaire items were positive (i.e. above the point of neutrality) and that the visitors strongly agree that they would recommend the system for heritage learning.

Six (6) participants highlighted the technology potential for learning in schools and museums. Eight (8) participants stated that the system enables them to better appreciate local (Perth) history by providing concrete, pictorial illustrations. Participants also appreciated the combination of history and technology in a museum setting.

Within the VR headset exhibit, some participants' suggested the inclusion of varied sound content for improved realism and as an alternate means of information delivery. Participants suggested the addition of ambient sound and audio narratives to make the experience more natural. There is evidence in literature to suggest that audio content affects users' perception of both the virtual and physical environments [ $4,5,6]$.

Structured interviews with museum curators highlighted conviction, cost and expertise as typical challenges to the adoption of digital exhibits in museum spaces and the following observations.

1. Museums may lack the conviction and may be skeptical about the use of digital exhibits owing to their perception of VR technology as a "work in progress". The museum adopted the digital exhibition in spite of this conviction because technology can serve as a "strong and interpretive tool" and has the potential to foster interaction and engagement with heritage.

2. The cost of equipment purchase and maintenance constitutes a challenge to the development of digital exhibits. However discussions reflected on how the reusability of digital content helps to spread out the cost of creating the content over time. For instance, the 3D content was used in the on-site exhibition, online virtual tours and mobile apps, thus demonstrating content reuse on multiple platforms which significantly reduces the effective-cost of content creation.

3. Museums may need to outsource technical tasks where the required expertise is not available in-house, as was the case with the Perth Museum and Art Gallery. However, outsourcing is not always feasible. Developing interdisciplinary collaborations and empowering museum staff can address this challenge. 
4. The availability of heritage experts is just as important as technical expertise. Museums may be short on manpower that possess the skills required to initiate technical projects and/or collaborate with technical experts. Awareness of museums' needs coupled with an allocation of resources can address this challenge.

As demonstrated by the survey data, the exhibition exceeded expectations from the visitors' perspective, as $82.30 \%$ thought it was good value for money and $90.43 \%$ expressed that they were either satisfied or very satisfied with the exhibition (see fig 7). The combination of physical and digital content enabled the museum to appeal to different audiences - those interested in novel technologies and those interested in traditional heritage content.

\section{Conclusion}

This paper has looked at the creative and technical development of a mixed reality exhibition, Picts and Pixels, which showed in the Perth Museum during the summer of 2017. From its early conception, this exhibition has brought together multiple modes of exploration of the past within a single gallery space. The physical and digital exhibits combined with the precisely planned atmosphere of the gallery came together to create an experience which took visitors back in time to an early Scotland. Through evaluation, the addition of immersive technology proved to greatly enhance the visitor experience; an undertaking that both helped imagine another time in history, as well as a group of people greatly misunderstood through time.

The exhibition raised and resolved important questions, including how to create a space where digital and physical exhibits complement each other as well as play on each other's strengths. We feel that overall we achieved this, primarily due to the parallel design of both components that was integrated from the beginning stages of planning. This allowed for the digital and physical to be complimentary tools to create an exhibition around a single and commonly mis-represented subject.

Picts and Pixels helped to normalize digital interpretation and virtual reality in a museum setting, treating them as instruments within the curator's toolkit that could be used together to create accessible, engaging and interactive exhibitions.

For all partners this project contributed to their long-term development and organisational resilience. It helped them to build audiences and brings the sharing of new expertise and knowledge, skills and resources in what was a genuinely new way of working for us. Using new technology to interpret collections and themes in an exciting and creative way helped reposition Perth Museum \& Art Gallery as an organisation that excites and innovates, offering visitors a creative experience combining traditional and digital interpretation. Lessons learned and increased staff skills contributed to and informed a major redevelopment of Culture Perth and Kinross venues, 2018-2022. For PKHT/TLP and the University of St Andrews Open Virtual Worlds it provides an opportunity to promote their work and develop the Museum in a Box toolkit for the sector. For local museum partners it brings closer working and co-operation and contribution to the Year of History, Heritage and Archaeology. xxxx xxxx xxxx xxxx xxxx xxxx xxxx 


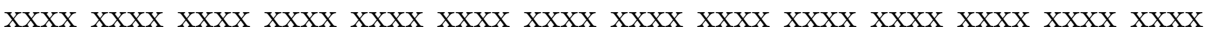
$\operatorname{xxxx} \operatorname{xxxx} \operatorname{xxxx}$

\section{References}

1. Wagner, P. and Konstam, A., 2012. Pictish Warrior AD 297-841. Bloomsbury Publishing. Vancouver

2. Keys, D., 2004. Rethinking the Picts. Archaeology, 57(5), pp.40-44.

3. Galani, A. and Chalmers, M., 2003. Far away is close at hand: shared mixed reality museum experiences for local and remote museum companions. Archives \& Museum Informatics, p.2.

4. Johnson, S. and Coxon, M., 2016. Sound can enhance the analgesic effect of virtual reality. Royal Society open science, 3(3), p.150567.

5. Orman, E.K., 2016. Effect of virtual reality exposure and aural stimuli on eye contact, directional focus, and focus of attention of novice wind band conductors. International Journal of Music Education, 34(3), pp.263-270.

6. Powell, W., Stevens, B., Hand, S. and Simmonds, M., 2010. Sounding better: Fast audio cues increase walk speed in treadmill-mediated virtual rehabilitation environments. Studies in health technology and informatics, 154, pp.202-207.

7. . Tait, E., Laing, R., Grinnall, A., Burnett, S. and Isaacs, J., 2016. (Re)presenting heritage: Laser scanning and $3 \mathrm{D}$ visualisations for cultural resilience and community engagement. Journal of Information Science, 42(3), pp.420-433.

8. Games methodologies and immersive environments for virtual fieldwork Getchell, K. M., Miller, A. H. D., Nicoll, J. R., Sweetman, R. J. and Allison, C. Oct 2010 In : IEEE Transactions on Learning Technologies. 3, 4, p. 281-293 13

9. . Exploring the Second Life of a Byzantine Basilica Getchell, K., Miller, A., Allison, C. \& Sweetman, R. J. 2009 Serious Games on the Move. Petrovic, O. and Brand, A. (eds.). Springer, p. 165-180

10. A virtual museum installation for virtual time travel Fabola, A. E., Kennedy, S. E., Miller, A. H. D., Oliver, I. A., McCaffery, J. P., Cassidy, C. A., Clements, J. and Vermehren, A. 7 Jun 2017 Immersive Learning Research Network: Third International Conference, iLRN 2017, Coimbra, Portugal, June 26-29, 2017. Proceedings. (Communications in Computer and Information Science (CCIS); vol. 725)

11. Exploring the past with Google Cardboard Fabola, A., Miller, A. and Fawcett, R. 28 Sep 2015 Proceedings of the 2015 Digital Heritage International Congress IEEE, Vol. 1, p. 277-284 8 p. 7413882

12. The Virtual Museums of Caen: a case study on modes of representation of digital historical content Miller, A., Vermehren, A. \& Fabola, A. 28 Sep 2015 Proceedings of the 2015 Digital Heritage International Congress IEEE, Vol. 2, p. 541-548 8 p. 7419571

13. Immersive Installation: "A Virtual St Kilda" Kennedy, S. E., Miller, A. H. D., Oliver, I. A., Watterson, A. \& Allison, C. 2015 Immersive Education: 4th European Summit, EiED 2014, Vienna, Austria, November 24-26, 2014, Springer, p. 101-113 (Communications in Computer and Information Science; vol. 486)

14. Exploring canons and cathedrals with Open Virtual Worlds: The recreation of St Andrews Cathedral, St Andrews Day, 1318 Kennedy, S. E., Fawcett, R., Miller, A. H. D., Sweetman, R. J., Dow, L., Campbell, A., Oliver, I. A., McCaffery, J. P. \& Allison, C. Oct 2013 Digital Heritage International Congress (DigitalHeritage), 2013 . IEEE, Vol. 2, p. $273-280$

15. Digitally enhanced community rescue archaeology Dawson, T., Oliver, I. A., Miller, A. H. D., Vermehren, A. \& Kennedy, S. E. Oct 2013 Digital Heritage International Congress (DigitalHeritage), 2013 . IEEE, Vol. 2, p. 29-36 
16. Edinburgh 1544 Video, S. Kennedy, Alan Miller, Catherine Cassidy, Smart History ltd https://vimeo.com/208677167

17. Exploring heritage through time and space: Supporting community reflection on the highland clearances, McCaffery, J. P., Miller, A. H. D., Kennedy, S. E., Dawson, T., Vermehren, A., Lefley, C. and Strickland, K. Oct 2013 Digital Heritage International Congress (DigitalHeritage), 2013 . IEEE, Vol. 1, p. 371-378 